\title{
Exciton localization in doped Si nanocrystals from single dot spectroscopy studies
}

\author{
I. Sychugov, ${ }^{1, *}$ J. Valenta, ${ }^{2}$ K. Mitsuishi, ${ }^{3}$ and J. Linnros ${ }^{1}$ \\ ${ }^{1}$ Department of Material Physics, School of ICT, Royal Institute of Technology, Electrum 229, Kista-Stockholm, 16440 Sweden \\ ${ }^{2}$ Department of Chemical Physics and Optics, Faculty of Mathematics and Physics, Charles University, Ke Karlovu 3, \\ Prague 2, 12116 Czech Republic \\ ${ }^{3}$ High Voltage Electron Microscopy Station, National Institute for Materials Science, Sakura 3-13, Tsukuba, 3050003 Japan
}

(Received 5 April 2012; revised manuscript received 5 June 2012; published 16 August 2012)

\begin{abstract}
The results of low-temperature photoluminescence characterization of single silicon nanocrystals prepared from highly doped silicon-on-insulator wafers are presented. The effect of B, P, As, and Sb impurities on ensemble as well as individual emission spectra are determined by comparison with the line shapes of undoped nanocrystals. From the statistical analysis of the luminescence spectra, the donor ionization energies for nanocrystals emitting in the range of $1.5-2.0 \mathrm{eV}$ are estimated to be 140-200 meV, while the exciton-impurity binding energy for Asand Sb-doped nanocrystals is found to be about $40-45 \mathrm{meV}$.
\end{abstract}

DOI: 10.1103/PhysRevB.86.075311

PACS number(s): 78.67.Bf, 78.55.Ap, 78.67.Hc, 61.72.uf

\section{INTRODUCTION}

Doping is known as an efficient way of controlling bulk semiconductor electrical properties and widely used for modern device fabrication. With ever decreasing device characteristic size, recent attention has been turned to the effect of doping in nanostructures. ${ }^{1,2}$ It is known that quantum size effects can substantially modify semiconductor properties at the nanoscale also affecting essential dopant characteristics, such as the ionization energy and Bohr radius. For example, in bulk silicon B, P, As, and Sb impurity levels are shallow. The ionization energy for the acceptor of group-III B is $45 \mathrm{meV}^{3}$ and for the donors of group-V P, As, and $\mathrm{Sb}$ are 44, 49, and $39 \mathrm{meV}$, correspondingly. ${ }^{4}$ However, it was calculated that these energy levels become deeper when the nanocrystallite size is approaching the quantum confinement regime..$^{5}$ This can be partly explained by a lowering of the effective dielectric constant due to the effects of confinement and the surrounding oxide matrix. Experimentally, this effect was observed for B-and P-codoped Si nanocrystals as a several hundred millielectron volt lowering of the luminescence peak energy in comparison to an undoped sample. ${ }^{6}$ In addition, by electron spin resonance, the donor size was estimated to be about half of that of a nanocrystal, scaling with size. ${ }^{7}$

In practice the presence of an impurity can be detected in the low-temperature photoluminescence (PL) spectrum by specific lines. ${ }^{8}$ In doped bulk material these new optical transitions originate from the exciton localization on a neutral impurity, denoted $\operatorname{as}^{0} X{ }^{0}{ }^{9}$ The binding energy of the exciton, according to the Haynes rule, is $\sim 10 \%$ of the impurity ionization energy. ${ }^{10}$ So in bulk silicon, which is an indirect band-gap material, such lines are located several millielectron volts below the virtually nonexistent no-phonon free exciton line. ${ }^{9}$ It is suggested that the localized impurity centers break the translational invariance of the crystal, making possible direct transitions without phonon mediation. ${ }^{11}$

When the impurity becomes ionized, on the other hand, the added free carrier is believed to lead to the nonradiative Auger process. $^{12}$ Recent calculations, however, indicate that the probability of this process strongly depends on the nanocrystal size. ${ }^{13,14}$ The reason lies in the discrete nature of energy states of a quantum dot. In some nanocrystals there are no final states for this resonant transition, and the radiative recombination channel can still prevail.

In this work doped Si nanocrystals were produced from highly doped silicon-on-insulator (SOI) wafers. They were probed by low-temperature PL, where individual and ensemble spectra were recorded. The advantage of the access to single nanocrystals is in avoiding inhomogeneity inherent to ensembles. It is used here for studies of dopants in nanostructures and effects of quantum confinement on their energy levels. The data were statistically analyzed and compared with previously reported PL results for low-doped wafers. ${ }^{15}$ Observed differences in optical emission patterns are discussed using the exciton localization model and available theoretical calculations.

\section{EXPERIMENTAL DETAILS}

Silicon nanocrystals were fabricated from thin SOI wafers with four different impurities by etching and short oxidation of the top silicon layer. Due to the thickness variation of the few nanometer-thin layers, in particular toward edges of the wafer, areas can be found in which local isolated silicon islands exist. The fabrication method is described in detail previously, ${ }^{16}$ although no lithographical patterning was employed here. The resistivity of wafers doped with $\mathrm{B}, \mathrm{P}$, As, and $\mathrm{Sb}$ atoms was selected to be $\leqslant 0.01-0.02 \Omega \cdot \mathrm{cm}$. It corresponds to a doping level of $\geqslant 5 \times 10^{18} \mathrm{~cm}^{-3}$, or on average one dopant atom per nanocrystal with a cube side of $6 \mathrm{~nm}$. So, some nanocrystals in these samples can randomly contain an impurity atom. Correspondingly, the luminescence fingerprint of a single particle is expected to differ depending on dopant incorporation. In the previous study nanocrystals were prepared from wafers with $15-25 \Omega \cdot \mathrm{cm}$ resistivity, which is 3 to 4 orders of magnitude higher. ${ }^{15}$ These luminescence spectra are used here for reference as undoped samples. Lowtemperature luminescence data were collected with a special wide-field cryo-microspectroscopy setup using a 405-nm laser diode for excitation of samples placed in a liquid He cryostat. ${ }^{17}$ A binned imaging mode, with reduced spectral resolution, was often selected in order to increase signal-to-noise ratio and for easier peak identification. For transmission electron microscope (TEM) imaging, focused ion beam (cross-section 

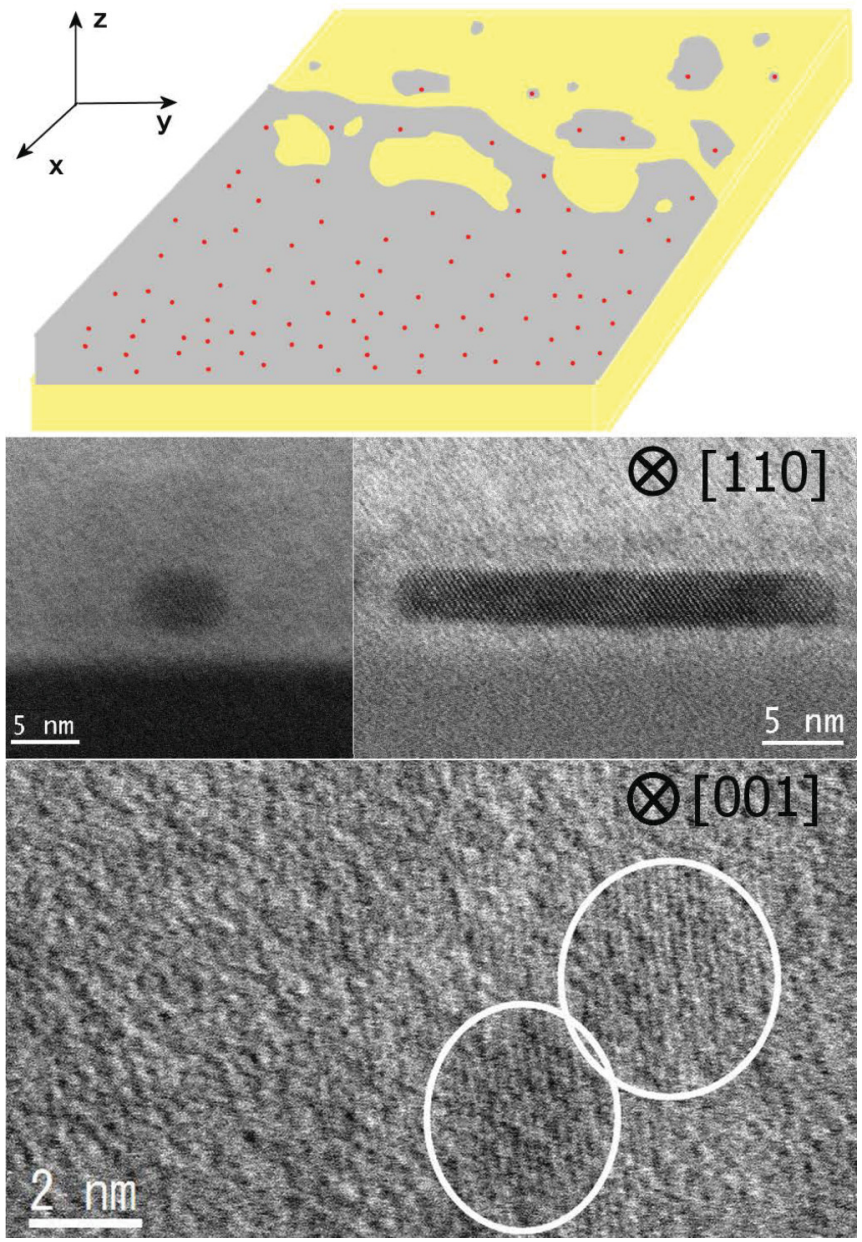

FIG. 1. (Color online) (Top) Schematic representation of an SOI chip with silicon (gray) layer on top of buried oxide [yellow (light gray)] doped with impurity atoms (red dots). The dry-etching process results in a layer thickness gradient, leading to the formation of a borderline region, where doped and undoped nanocrystals as well as large nanoislands of silicon are formed. (Middle) Bright-field highresolution cross-sectional TEM images of such a region confirming the existence of Si quantum dots (left) and quantum wells (right) on top of the buried oxide (bottom). A top-view high-resolution TEM image with visible Si lattice fringes of two nanocrystals, highlighted with circles.

view) and dimpling (top-view) preparation techniques were used.

In Fig. 1 (top) a schematic representation of the etched SOI wafer with a Si layer thickness gradient is shown. The region on the sample between exposed buried oxide and remaining silicon film contains random silicon nanostructures with and without dopant incorporation. This part of the sample can be easily identified in the reflected white-light image by color and then targeted in PL measurements. Below on the same figure, TEM images from this region are presented. On the high-resolution cross-sectional image taken along the [110] direction, individual Si quantum dot (left) and quantum well (right) nanostructures on top of buried oxide are shown. The high-resolution image taken in the top-view geometry shows two closely spaced silicon nanocrystals (Fig. 1, bottom). The exciton Bohr radius in silicon is about $5 \mathrm{~nm}$, so the
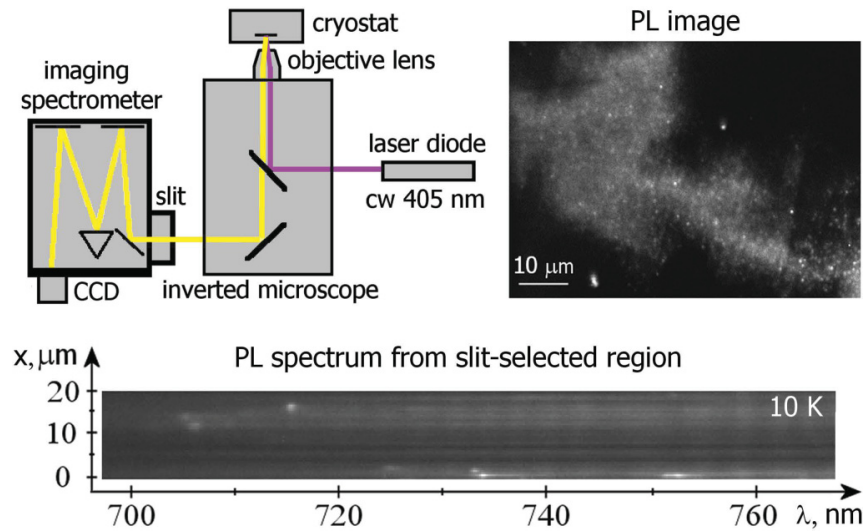

FIG. 2. (Color online) (Top, left) Schematic view of the experimental setup for the PL measurements. (Top, right) PL image of the borderline region with silicon nanostructures. (bottom) Lowtemperature PL spectrum of the slit-limited $20-\mu \mathrm{m}$-long stripe from the borderline region. It contains sharp spectra from individual nanocrystals at different emission wavelength as well as a broad ensemble spectrum.

images confirm the presence of nanostructures with threeand one-dimensional quantum confinement in the area of interest.

The scheme of the PL setup for optical characterization is given in Fig. 2 (left). A typical PL image of the borderline region, where individual emitters can be discerned on top of the uniform background, is shown for the As-doped sample (Fig. 2, right). Dark areas below and above the luminescent part correspond to the regions without the remaining silicon and with too thick a layer for confinement effects to manifest. For spectroscopic characterization the signal from a slit-limited region was dispersed in the spectral domain by gratings of the imaging spectrometer and recorded by the charge-coupled device (CCD) detector. An example of such a spectral image is shown in Fig. 2 (bottom). One can distinguish sharp luminescence lines positioned at different emission wavelengths. Such narrow luminescence linewidths and various emission energies are characteristic features of individual quantum dots, originating from their discrete energy levels and quantum size effects. Thus, it is possible here to clearly resolve low-temperature luminescence signal from single nanocrystals. In particular, on this image there are six peaks, where the bottom spectrum has a replica $\sim 19 \mathrm{~nm}$ (42 meV) apart. An analysis of replica positions in comparison with undoped nanocrystals is provided below. In order to exclude possible contribution of other emitters to the same spectral line, a statistical approach was employed here with more than 1000 individual spectra recorded and analyzed.

In addition to narrow spectra from individual emitters, we also detect very broad lines, which are temperature independent. On the spectral image in Fig. 2 (bottom) such a spectrum can be seen in the top part, spanning tens of nanometers. These bands most likely originate from different kinds of emitters located within the same diffraction-limited spot, seen as a uniform background on the PL image. A combination of luminescence from quantum wells and nanocrystals of various sizes can result in the observed broad ensemble spectrum. 


\section{RESULTS}

In order to additionally confirm the single emitter nature of luminescence, we performed a set of time sequence measurements for the sharp peaks. In Fig. 3 time dynamics of two different narrow lines is shown as a continuous set of ten spectra with 5-min integration time for each frame. Here, typical features of a single emitter, such as spectral diffusion and blinking, are represented. The former effect (Fig. 3, left) is believed to originate from the fluctuation of the electric field in the vicinity of a nanocrystal. ${ }^{18}$ The accumulation and dissipation of charges close to the nanocrystal emitter can be responsible for the observed dynamic shift of the peak position within several millielectron volts analogous to the Stark effect. This stochastic process contributes to the broadening of the nanocrystal luminescence linewidth in a time-integrated experiment, where these fluctuations are summed up. In addition to the spectral diffusion, we have also observed "spectral hopping" in emission from some nanocrystals, where the peak position abruptly shifts several tens of millielectron volts and back in a reversible process. It is not clear at this point if such spectral "jumps" are caused by a stronger Stark shift or intrinsic recombination in the nanocrystal.

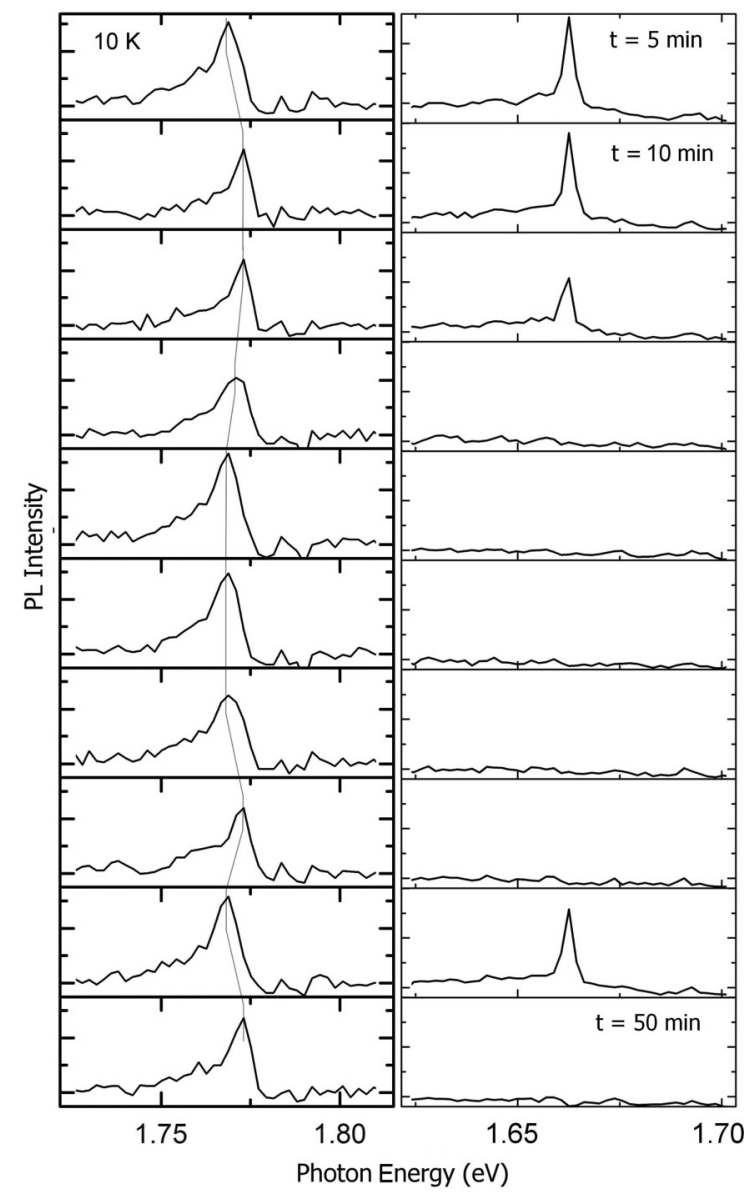

FIG. 3. Time dynamics of two different individual luminescence spectra showing (left) spectral diffusion and (right) blinking effects. Each frame integration time was $5 \mathrm{~min}$.

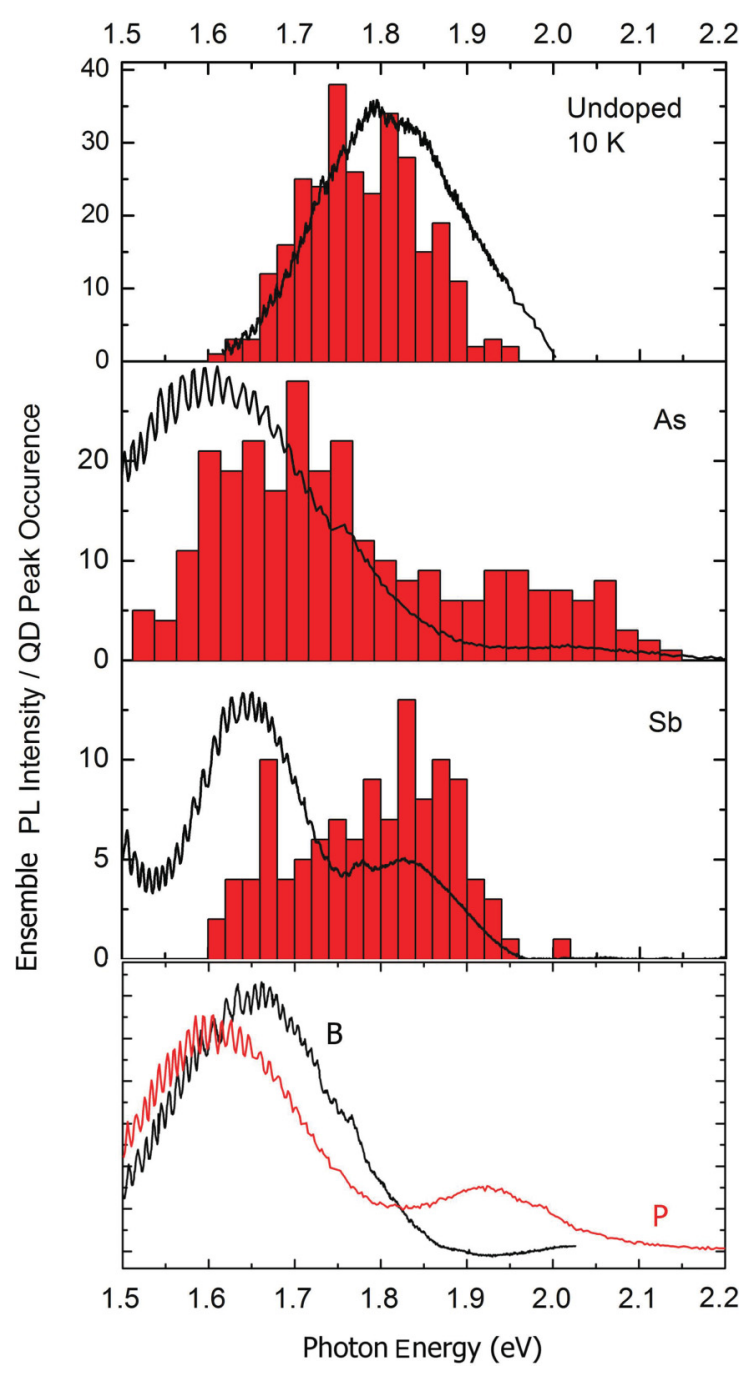

FIG. 4. (Color online) Ensemble spectra of undoped and differently doped silicon nanostructures (solid lines); fringes are from the etaloning effect of a back-thinned CCD camera. Histograms of peak position occurrence for individual quantum dots (QDs) for undoped (top), As- (middle), and Sb-doped (bottom) samples.

The random intermittence of luminescence (Fig. 3, right), or switching between "on" and "off" states, can also be attributed to nanocrystal environment. ${ }^{19}$ It is believed that the excited carrier trapping in the surrounding matrix makes possible nonradiative Auger process inside the nanodot, quenching the light emission. The light-emitting period resumes when the initial conditions are restored by charge detrapping, resulting in the observed blinking behavior.

In Fig. 4 ensemble spectra are plotted for all four doped samples as well as for the undoped one. The main peak for all samples with impurities is shifted to the lower-energy side compared to the undoped sample. The smallest shift is recorded for the B-doped sample $(\sim 140 \mathrm{meV})$ and the largest for As- and P-doped ones ( $\sim 200 \mathrm{meV})$. For each dopant there is also a new, smaller band appearing at the high-energy side of the spectrum. Histograms of the peak position occurrence for individual nanocrystal spectra are also shown for undoped and As- and Sb-doped samples. These distributions mainly follow the curve of the corresponding ensemble spectra, except for 

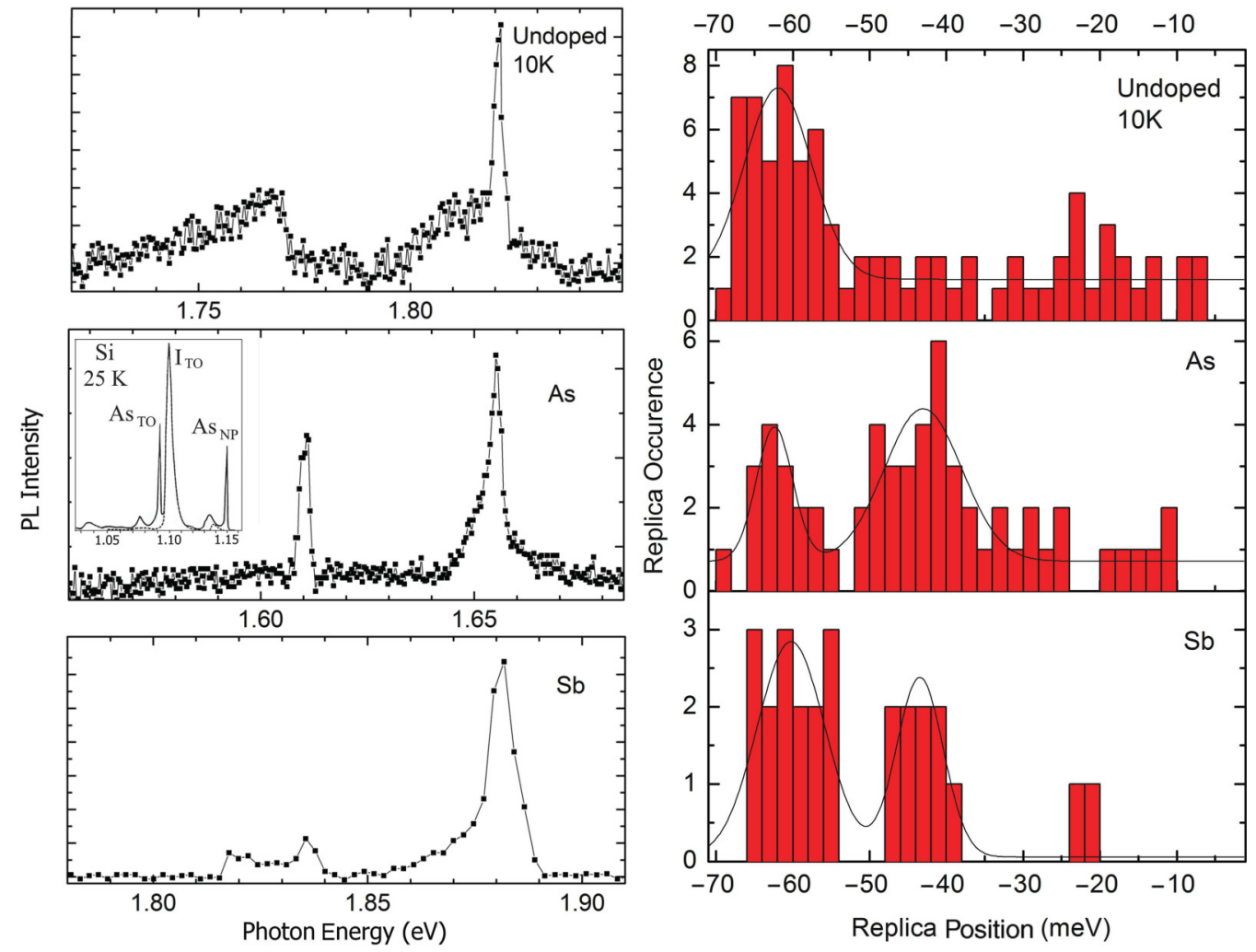

FIG. 5. (Color online) (Left) Three representative low-temperature spectra of undoped, As-doped, and Sb-doped Si nanocrystals. Sb-doped spectrum was acquired using lower spectral resolution grating. Inset on the second figure shows PL lines from As-doped bulk Si. (Right) Statistical distribution of the replica occurrence in relation to the main peak position for these three samples. While spectra with TO-phonon sidebands $(\sim 60 \mathrm{meV})$ exist in all samples, some nanocrystals in As- and Sb-doped samples exhibit a new feature at $\sim 40-45 \mathrm{meV}$. The solid lines are guides to the eye.

the low-energy side. The presence of other radiation sources, such as quantum wells, in the ensemble spectra may produce a low-energy signal that would not have corresponding quantum dots.

In general, according to the quantum confinement model, the emission from Si nanocrystals with energy 1.7-1.8 eV (peak of distribution in Fig. 4) corresponds to 3- to 4-nmdiameter particles. ${ }^{20}$ From Fig. 1 one can see that nanocrystals investigated here have a spherical shape. So the doping concentration $\geqslant 0.005$ atoms $/ \mathrm{nm}^{3}$ translates to $\geqslant 0.1$ dopant per nanocrystal on average.

Examples of typical single spectra for undoped and As- and Sb-doped samples are given in Fig. 5 (left). The structure of individual spectra and their emission energy distribution for the doped samples appeared to be similar to the undoped ones. The line shape is dominated by the main peak accompanied by sidebands at the lower-energy side. The main narrow line is ascribed to zero-phonon recombination of quantum confined carriers in a nanocrystal. ${ }^{15}$ All the samples contain some nanocrystals exhibiting replica at $\sim 60-\mathrm{meV}$ distance from the main peak. It is attributed to the TO-phonon-assisted process, inherited from the indirect band-gap bulk material. In addition, the As- and $\mathrm{Sb}$-doped samples have some spectra revealing a feature located $\sim 40-45 \mathrm{meV}$ below the no-phonon line. A spectrum of As-doped bulk silicon, composed of no-phonon and TO-phonon-bound exciton lines $\mathrm{As}_{\mathrm{NP}}, \mathrm{As}_{\mathrm{TO}}$ as well as a free exciton phonon line $\mathrm{I}_{\mathrm{TO}}$, is provided for reference on the inset. $^{8}$ To exclude possible contribution from other emitters to the same spectral line, statistical analysis of the interpeak spacing was carried out. It is given in Fig. 5 (right), with only one maximum visible in this distribution for undoped samples and two for As- and Sb-doped samples. Note that all spectra of doped samples would not exhibit extra peaks since nanocrystals may, statistically, be undoped. No specific features except for the TO-phonon line were systematically detected for B- and P-doped nanocrystals.

\section{DISCUSSION}

First, we note that the shift in the ensemble spectra from the undoped spectra position by $140-200 \mathrm{meV}$ is consistent with theoretical calculations of the energy level positions of $B$ and $\mathrm{P}$ impurities in such nanocrystals. ${ }^{5}$ The separation of the acceptor level from the top of the valence band for boron is calculated to be about $100 \mathrm{meV}$, while the phosphorus donor level is expected to lie $\sim 200 \mathrm{meV}$ below the bottom of the conduction band. The ensemble spectra contain emission from various constituents, and in some of them the recombination may proceed via the impurity levels, reducing the emission energy by the value of the level band separation.

The analysis of individual spectra shown in Fig. 5 (right) reveals a new feature in As- and Sb-doped samples. For the former the statistical distribution of the separation between two adjacent peaks has two maxima on a somewhat uniform 
background level. The peaks from different objects contributing to the same spectral line are expected to be randomly separated in the spectral domain, adding pedestal to this distribution. On the other hand, TO-phonon and $\sim 40-45 \mathrm{meV}$ replicas are often encountered in these spectra, resulting in two clear maxima. The statistics of Sb-doped nanocrystal spectra is somewhat worse, but the distribution looks similar to the As-doped case. This new spectral feature can coexist with the TO-phonon line (Fig. 5, left, bottom) or can be a stand-alone sideband (Fig 5, left, middle). We attribute this line to the exciton bound to a neutral donor in a nanocrystal, $\mathrm{As}^{0} X$ and $\mathrm{Sb}^{0} X$. The binding energy of $\sim 40-45 \mathrm{meV}$ differs significantly from the value $\sim 5 \mathrm{meV}$ in the bulk material. ${ }^{9} \mathrm{We}$ also note that $\sim 12 \%$ of the individual spectra possess this new feature, which is comparable to the $10 \%$ estimated fraction of doped nanocrystals.

The empirical Haynes rule, relating exciton binding energy as $\sim 0.1$ of the dopant ionization energy, is expected to change at the nanoscale. ${ }^{21}$ It was calculated that it is about two times higher for quantum wells and can be even larger for zero-dimensional nanostructures. Assuming its value of 0.25 for Si nanocrystals, the position of the observed bound exciton replicas $\sim 40-45 \mathrm{meV}$ would translate to 160 - to $180-\mathrm{meV}$ deep impurity levels. These values are very similar to the observed ensemble shifts in the luminescence peak position for As- and Sb-doped samples, 200 and $160 \mathrm{meV}$, correspondingly (cf. Fig. 4). It should be noted that As and Sb are neighboring elements of the group $\mathrm{V}$ and can be considered as heavy impurities in silicon. So the exciton-impurity interaction in Si nanostructures can be expected to be similar for these two elements.

Low-temperature PL studies of doped bulk material reveal only weak binding of the exciton to boron. ${ }^{8}$ The $\mathrm{B}^{0} X$ line intensity value of $0.005 \mathrm{I}^{\mathrm{TO}}$, where $\mathrm{I}^{\mathrm{TO}}$ is the TO-assisted line strength, is the weakest among the dopants investigated here. At the same time the intensity of the strongest excitonimpurity complex, $\mathrm{As}^{0} \mathrm{X}$, is more than 2 orders of magnitude larger: $0.8 \mathrm{I}^{\mathrm{TO}}$. In low-dimensional silicon structures, boron is also not expected to enhance the zero-phonon transition rate. $^{22}$ This is believed to be due to the lack of a short-range field capable of the efficient band mixing, which cancels the $k$-conservation rule of the bulk material in nanostructures. So the absence of new prominent features in the low-temperature spectra of individual B-doped nanocrystals would be expected. On the other hand, phosphorus has a strong $\mathrm{P}^{0} X$ peak in bulk $\mathrm{Si}$ and was predicted to promote radiative recombination in nanocrystals. ${ }^{22}$ It should be investigated further if the lack of observed features in the spectra of P-doped nanocrystals here is due to insufficient statistics.

\section{CONCLUSIONS}

To summarize, silicon nanocrystals emitting in the 1.5 $2.0 \mathrm{eV}$ spectral range and doped with four different impurities were probed by the low-temperature micro-PL technique. A large number of individual spectra were recorded and analyzed. Differences in ensemble spectra for the B-, P-, As-, and $\mathrm{Sb}$-doped samples and in single spectra for As- and $\mathrm{Sb}$ doped samples, compared to the undoped one, were detected. In addition to the TO-phonon line, we observed a new line, shifted by 40-45 meV from the no-phonon peak in As- and Sbdoped nanocrystals, attributed to an impurity localized exciton. The exciton binding energy for As- and Sb-doped nanocrystals was estimated to be an order of magnitude larger than in bulk silicon, while the energy level positions for these dopants were found to be several times deeper than in bulk material. Further investigation is needed for deeper understanding of the dopant levels and their influence on the excited carrier relaxation and recombination, which is fundamental for applications of $\mathrm{Si}$ nanocrystals in, for example, solar cells. ${ }^{23}$

\section{ACKNOWLEDGMENTS}

The support from the Swedish Research Council (VR) and Japan Ministry of Education, Science, Sports and Culture (MEXT) through "Nanotechnology Network Project" is acknowledged. Part of the work was done under the project NASCEnT funded by the EC FP7 program (GA No. 245977).
*Corresponding author: ilyas@kth.se

${ }^{1}$ D. J. Norris, A. L. Efros, and S. C. Erwin, Science 319, 1776 (2008)

${ }^{2}$ D. Mocatta, G. Cohen, J. Schattner, O. Millo, E. Rabani, and U. Banin, Science 332, 77 (2011).

${ }^{3}$ E. Burstein, E. E. Bell, and J. W. Davisson, J. Phys. Chem. 57, 849 (1953).

${ }^{4}$ F. J. Morin, J. P. Maita, R. G. Shulman, and N. B. Hannay, Phys. Rev. 96, 833 (1954).

${ }^{5}$ E. L. Ramos, E. Degoli, G. Cantele, S. Ossicini, D. Ninno, J. Furthmuller, and F. Bechstedt, J. Phys.: Condens. Matter 19, 466211 (2007).

${ }^{6}$ M. Fujii, Y. Yamaguchi, Y. Takase, K. Ninomiya, and S. Hayashi, Appl. Phys. Lett. 85, 1158 (2004).

${ }^{7}$ M. Fujii, A. Mimura, S. Hayashi, Y. Yamamoto, and K. Murakami, Phys. Rev. Lett. 89, 206805 (2002).
${ }^{8}$ I. Pelant and J. Valenta, Luminescence Spectroscopy of Semiconductors (Oxford University Press, Oxford, 2012), Chap. 7.

${ }^{9}$ P. J. Dean, J. R. Haynes, and W. F. Flood, Phys. Rev. 161, 711 (1967).

${ }^{10}$ G. Davies, J. Lumin. 80, 1 (1999).

${ }^{11}$ P. Y. Yu and M. Cardona, Fundamentals of Semiconductors: Physics and Materials Properties (Springer-Verlag, Berlin, 2001), p. 194.

${ }^{12}$ V. A. Kharchenko and M. Rosen, J. Lumin. 70, 158 (1996).

${ }^{13}$ G. E. Gragg and A. L. Efros, Nano Lett. 10, 313 (2010).

${ }^{14}$ N. V. Kurova and V. A. Burdov, Semiconductors 44, 1414 (2010).

${ }^{15}$ I. Sychugov, J. Valenta, K. Mitsuishi, M. Fujii, and J. Linnros, Phys. Rev. B 84, 125326 (2011).

${ }^{16}$ I. Sychugov, Y. Nakayama, and K. Mitsuishi, Nanotechnology 21, 285307 (2010). 
${ }^{17}$ J. Valenta, B. Bruhn, and J. Linnros, Nano Lett. 11, 3003 (2011).

${ }^{18}$ S. A. Empedocles and M. G. Bawendi, J. Phys. Chem. B 103, 1826 (1999).

${ }^{19}$ B. Bruhn, J. Valenta, F. Sangghaleh, and J. Linnros, Nano Lett. 11, 5574 (2011).

${ }^{20}$ G. Ledoux, J. Gong, F. Huisken, O. Guillois, and C. Reynaud, Appl. Phys. Lett. 80, 4834 (2002).

${ }^{21}$ J. Singh, Phys. Solid State 40, 728 (1998).
${ }^{22}$ V. A. Belyakov and V. A. Burdov, Phys. Rev. B 79, 035302 (2009). ${ }^{23}$ S. Janz, P. Löper, M. Schnabel, M. Zacharias, D. Hiller, S. Gutsch, A. M. Hartel, C. Summonte, M. Canino, M. Allegrezza, S. Ossicini, R. Guerra, I. Marri, B. Garrido, S. Hernández, J. López-Vidrier, J. Valenta, T. Kubera, M. Foti, and C. Geradi in Proceedings of the 26th European PV Solar Energy Conference \& Exhibition, Hamburg, 2011 (WIP-Renewable Energies, Munich, 2011), pp. 22-27. 